\title{
Las reformas del Estado, de las políticas sociales y de salud. Un balance del final del milenio
}

\author{
Public sector and social and health policy reforms. \\ An inventory on the eve of the new millennium
}

Silvia Gerschman 1

\footnotetext{
1 Departamento de Administração ePlanejamento em Saúde, Escola Nacional de Saúde Pública, Fundação Oswaldo Cruz. Rua Leopol do Bulhões 1480, Rio deJaneiro, RJ 21041-210, Brasil.
}

A bstract This study reflects on reforms in health systems and social policies within the framework of the so-called public sector reforms. The point of departure is a review of various explanations for the crisis in theWel fare State, present in the literature from the 1990s. Social policies, at the heart of the crisis, are heavily challenged. What we intend to demonstrate is that this argument plays a specific role, that of introducing neoliberal changes into economic policy, in which the economic tool s u sed generate abstention by the statefrom the social sphere, deregulation of national economi es in favor of the free market, and the fundamentally oversized role of the international financial market. Within this context we analyze the social security and heal th system reforms. The final part of the article deals with current difficulties in social policies, focusing the debate on a mapping of possiblealternatives for devel oping social and health policies. Key words Health System; Health Policy; Public Policy

Resumen El presente trabajo se propone refl exionar sobre las reformas de los sistemas de salud y de pol íticas social es en el marco delas así llamadas reformas del Estado. Com esta finalidad, se busca como punto de partida condensar distintas visiones explicativas de la crisis del Estado de Bienestar, presentes en la bibliografía produzida en la década de los 90. En el corazón de la crisis, las políticas sociales se encuentran profundamente cuestionadas. Lo que pretendemos mostrar es que este argumento posee una función específica, que es la de introducir mudanzas de carácter neoli beral en la política económica y para las cuales los instrumentos económi cos utilizados van a generar la abstención del Estado en el plano social, la desregulación de las economías nacional es en favor del libre mercado y, fundamentalmente, el papel sobredimensionado del mercado financiero internacional. En este contexto, son anal izadas las reformas de la seguridad social y de los sistemas de salud. La última parte del trabajo tuvo por objetivo enfrentar las dificultades que las políticas sociales presentan en el contexto actual, trayendo al centro del debate un mapeamiento de las alternati vas posibles para el desarrol lo de las políticas social es y desal ud. Palabras clave Sistemas de Salud; Políticas de Salud; Políticas Sociales 


\section{La crisis del Estado de Bienestar}

Reflexionar al respecto de las reformas de los sistemas de salud y de políticas sociales en los países del continente nos exige un esfuerzo de contextualizarlas en el marco de las así ll amadas reformas del Estado. Con esta finalidad, se hace necesario comenzar el trabajo por una discusión que nos permita comprender cómo surge y qué se entiende por Reforma del Estado.

Se trata de una idea históricamente fecha$\mathrm{da}$, fines de los años 70 , cuando sucesivas crisis económicas del capitalismo mundial, resultantes en poderosas deudas internas y externas, acompañadas de la pérdida del valor de monedas fuertes en los países centrales y del crecimiento de procesos inflacionarios en la mayoría de las economías, comprometieron la estabilidad y la sobrevivencia de los Estados de Bienestar Social europeos. Así, las conquistas de la clase operaria y la ascensión de los partidos social-demócratas que sustentaron el WelfareState se vieron considerablemente amenazadas, de una parte, por las tendencias económicas pragmáticas de sal ida de las condiciones adversas de las economías mundiales; y, de otra, por el avance de ideologías neoliberales que atribuyen al creciente papel del Estado en la regulación económica y en las políticas de seguridad social el motivo principal de los males que aquejan al capitalismo.

La bibliografía sobre la Reforma del Estado en los años 90 refleja y busca explicar la crisis del Estado de Bienestar y las salidas de la misma sobre diversas perspectivas. Los enfoques que abordan la crisis del Estado, como el resultado de las dificultades que se presentan para la "gobernabilidad" del mismo, enfatizan la incapacidad del Estado para administrar demandas sociales, considerando que la ampliación de los beneficios sociales a toda la población se transformó en un derecho de los ciudadanos, en cuanto los recursos se tornaron cada vez más escasos.

Sin embargo, entre los autores que explican la crisis del Welfare Statepor el crecimiento del papel social del Estado, algunos interpretan que la crisis del Estado es una crisis de crecimiento, consecuentemente positiva, y en este sentido es necesario racionalizar y reorganizar el Estado sin que esto signifique un achicamiento del mismo. En cuanto otros sostienen que el Estado creció desmedidamente en desacuerdo con el papel que le cabe, debiendo ahora abstenerse de intervenir en lo social, ya que cada individuo debe ser proveedor de su propio bienestar.
En los enfoques que consideran que la crisis del Estado se debe, principalmente, al incremento de los gastos sociales que resultan en el crecimiento desmesurado de las deudas públicas, las consideraciones que se tejen sobre este fenómeno divergen considerablemente, según el lugar del espectro político en el cual los autores se sitúan. Para al gunos de estos autores que se encuadran en una visión más societaria, se origina una crisis de legitimidad del Estado de Bienestar, debido al aumento de los impuestos que resultan onerosos a los ciudadanos, sin una contrapartida en término de las mejorías en la cantidad y calidad de los beneficios recibidos. Dentro de esta línea de pensamiento, en parte de la bibliografía existente, se caracteriza que el crecimiento desmedido del Estado se localiza en el papel de empleador, que ocupa una burocracia inoperante y que genera una ineficiencia cada vez mayor. Al mismo tiempo que se crea una nueva casta social de tecnócratas y burócratas, cuya sobrevivencia depende de un Estado enfermo de gigantismo e inmovilismo.

Cada vez más un mayor número de funcionarios atienden un menor número de personas, generando una multiplicación de programas sociales para estratos cada vez menores de la población que, en lugar de generar solidaridad social, crean descontentamiento en amplios sectores de la población. De una parte, el desempleo creciente va marginalizando sectores sociales cada vez mayores, que no se encuentran favorecidos por estos programas encaminados a minorías social es. De otra parte, para atender estos programas, se emplea una burocracia cada vez más numerosa erogando considerables recursos del Estado en el pago de los salarios de funcionarios públicos. Para visiones de carácter neoliberal éste sería el motivo para acabar con el Estado de Bienestar, quitándole cualquier función social y transformán dolo en un órgano mínimo.

A propósito de las políticas sociales: política vs economía en el contexto de la globalización

El papel regulador del Estado en la economía, incentivando el crecimiento económico, el pleno empleo, el consumo y la demanda de bienes, sustentado por el keynesianismo, y el creciente intervencionismo por la vía de implementación de políticas sociales que neutralicen las desigualdades sociales que el mercado "natural mente" crea, fueron los ejes centrales de la política económica que origi nó los Esta- 
dos de Bienestar europeos, sin al terar estructuralmente el modo de producción capitalista. Mas, al volver la mirada para Marx en su Crítica a la Economía Política, constatamos que es en el proceso de producción de mercancías que se genera el valor excedente, el lucro del capital que es capaz de ampliar y reproducir el proceso de producción capitalista, siendo, por lo tanto, el pilar que sustenta la modalidad de producción capitalista.

Pero, ¿qué ocurre cuando el lucro del capital reinvertido en la producción comienza a tener tasas decrecientes, sea por los avances tecnológicos, sea por las conquistas de los trabajadores a partir de las luchas sociales crecientes de las últimas décadas? Se produce un desplazamiento del capital productivo para las inversiones financieras que garanticen lucros mayores y más rápidos. La sobrevaloración del mercado financiero y la concentración fabulosa del capital, como pilares de las economías capitalistas de las dos últimas décadas, adquirieron alas doctrinarias, con el liberalismo tardío transformándose en la filosofía económica y política del fin del milenio.

En verdad, es en el mercado que se realiza la plusvalia, ya que, si bien en el proceso de producción se concreta la apropiación del trabajo excedente, esta apropiación se torna efectivamente lucro en el mercado. Pero, ¿qué ocurre cuando en el mercado no se realiza el lucro que deviene del trabajo excedente en el proceso productivo? Otras formas complementarias a la producción y al intercambio de mercancías en el mercado se tornaron predominantes para extraer lucro del capital a partir de los años 70.

La creación progresiva de instrumentos de política económica tornó el propio dinero en una mercancía capaz de generar lucros crecientes no comparables a los lucros obtenidos en la esfera productiva. Dando a los mercados financieros un lugar privilegiado en las economías capital istas e incentivando instrumentos de política económica, generadores de economías centradas en la especulación financiera y en el favorecimiento de la asociación trasnacional del capital - la globalización de la economía - por la vía de la desregulación de las economías nacionales.

La creciente disminución del papel regulador del Estado sobre los lucros del capital se expresó a través de medidas de liberalización económica, entre las cuales vale mencionar el aumento de las tasas de interés para el capital que entra en el circuito financiero, sin que el Estado desarrolle un papel específico, ya sea creando incentivos especiales u otros estímulos para promover la inversión productiva. En cuanto el Estado había tenido, en décadas pasadas, un papel político de condensar y/ o sintetizar relaciones entre diversas fuerzas económicas y así ejercía un papel decisorio en el arbitraje entre intereses sociales en pugna; en el presente, el Estado pierde ésta y cualquier función que signifique algún tipo de interferencia con los movimientos del capital globalizado.

Una cita de François Chesnais, sobre las afirmaciones, en un debate público, de uno de los mayores grupos económicos europeos, ilustra de manera elocuente la discusión enfrentada encima:

“...I a mundialización del capital da libertad a los grupos económi cos de impl antarse donde prefieran,para producir lo que prefieran,abasteciéndose y vendiendo donde prefieran, como así también soportando el mínimo posible de obl igaciones en materia dederechos de trabajo y de seguridad social" (Chesnais, 1997:9).

En este sentido, al analizar la globalización económica, Delcourt (1992) afirma que, en la medida que estos conjuntos de emprendimientos y recursos se transnacional izan, escapan a las normas nacionales y se sustraen al control político y democrático, tales como formas nacionales de concertación y de negociación entre partes o interlocutores sociales.

En una misma dirección, aunque dentro de una visión política del papel del Estado en el contexto de la globalización, Diniz (1998) destaca que la valoración de la dimensión política es fundamental para la eficacia de la acción estatal. La actuación estatal no depende solamente de la capacidad de tomar decisiones, pero sobretodo de las estrategias políticas de implementación de las decisiones.

El éxito de las políticas gubernamentales requiere, además de los instrumentos institucionales y de los recursos financieros controlados por el Estado, la mobilización de los medios políticos de ejecución. Garantizan la viabilidad política; esto significa tomar en cuenta la capacidad de articular alianzas y coaliciones que den sustentabilidad a las políticas gubernamentales, cualquiera que sea el régimen político que esté en vigor (Grindle \& Thomas, 1991; Silva, 1993).

Si bien Diniz pone el énfasis en la valoración de la dimensión política, el problema reside en que la construcción de alianzas entre intereses diversos y consecuentemente conflictuantes implica ceder una parte del poder decisorio y de execución para aquellas representaciones políticas (sociales y/ o partidarias) que no se enquadran en las modalidades tradicionales del ejercicio del poder político por parte de las élites locales. En el caso de los estados 
latinoamericanos, es precisamente aquí que se frena cualquier propuesta de avance democrático.

En este sentido, las trabas para el ejercicio de la acción política al interior del Estado en América Latina se apoyan sobre un tripé conformado por: el movimiento del capital transnacionalizado, los principios que rigen las economías y las agencias económicas mundiales y las trayectorias políticas nacionales. Estas últimas, en el caso de América Latina, son profundamente marcadas, de una parte, por la ausencia de la ciudadanía y la presencia de diferencias social es extremas, y, de otra, por la permanencia de un poder político autocrático en manos de las élites locales.

Así, el Estado pasa a ser el objetivo de la globalización económica en el continente, pasando a ser la Reforma del Estado - léase Estado mínimo - la única política plausible a ser seguida por las élites locales para disminuir el déficit fiscal, en acuerdo con la exigencia sine qua non de las agencias internacionales - FMI/ Banco Mundial - con los países del continente, para apoyar el refinanciamiento de los pagos de las deudas externas de estos países.

Junto a la pérdida de un papel más decisivo del Estado, se esfuma también cualquier esperanza de corrección de las enormes desigualdades sociales; las ya existentes más aquellas que la propia política económica crea a través de la recesión y del desempleo. Al mismo tiempo, se difunde y se extiende la idea de la inoperancia del Estado para encaminar políticas sociales, ya que éste no es su papel y, precisamente porque, por haberse desviado del mismo, creció en número de funcionarios y en el monto de los gastos. Así, si el Estado está condenado a ser ineficiente, la salida "natural" es su paulatina desaparición y no la manera de tornarlo eficiente.

Consecuentemente, se trata de una discusión que, lamentablemente, como vimos, no se originó en las enormes dificultades históricas de los estados latinoamericanos de satisfacer de manera resolutiva y equitativa Ias necesidades de salud, educación, vivienda, saneamiento, originadas por la pobreza creciente de nuestras sociedades. Por el contrario, de lo que se trata es de tornarlo cada vez más ineficiente. Si el Estado se transforma en una máquina sobredimensionada, cara e ineficiente, los argumentos que consideran al Estado como um espacio primordial para la distribución de la justicia social dejan de tener credibilidad.

Digo lamentablemente porque, en los gobiernos latinoamericanos, si el esfuerzo de reflexionar sobre el papel del Estado estuviese puesto en las posibilidades, que no se presentan con facilidad, pero que habría que indagar, a través de las brechas del proceso de globalización de la economía, tal vez, se podría alcanzar un diagnóstico más preciso de los problemas, de las intervenciones políticas y de los recursos necesarios para ser disponibilizados por los órganos gubernamentales - Ministerios de Previsión Social, Trabajo, Salud, Educación, Vivienda, etc. - de una manera más apropiada que aquella que el "ajuste" neoliberal propone.

Hoy, en lugar de subsanar los mal es que la pobreza crea en nuestras sociedades, el problema central se desplazó para las dificultades de financiamiento para el área social, independientemente del crecimiento de las necesidades que las formas tradicionales de pobreza presentan, sumadas a la marginalización y a la exclusión social, resultantes de las políticas de ajuste. Así, la reducción del Estado se presenta como la única manera de resolver las crecientes deudas fiscales y externas, manteniendo la estabilidad de las monedas.

Dos cuestiones se colocan como ejes de análisis del contexto actual de la reformas:

a) Ia enorme deuda social de las últimas décadas fue resultado de políticas no redistributivas de la riqueza produzida por la sociedad y de la responsabilidad atribuible a los gobiernos latinoamericanos por la dinámica imprimida en el ejercicio de la política pública.

La modalidad prevaleciente de actuación del Estado generó un crecimiento desmedido del mismo, creando una burocracia ineficiente y mal remunerada, transformando la esfera pública en dominio privado, favoreciendo sectores históricamente privilegiados de la sociedad, con el predominio de prácticas de clientelismo político y corrupción.

Esta opción política distante de la sociedad fue acompañada de las crisis económicas que se hicieron sentir desde los comienzos de los 80. Así pueden detectarse serios problemas de gobernabilidad del Estado. Entendemos que los problemas de gobernabilidad del Estado se situan en la dificultad de gerenciar las propias funciones del gobierno. En el momento que éstas enfrentan el crecimiento del desempleo, de la pobreza y de la marginalización social, junto al aumento de demandas sociales por políticas más ecuánimes, en un contexto adverso de crecimiento de la deuda pública interna y externa. Podríamos caracterizar este proceso como um movimiento endógeno y vicioso del Estado en América Latina, en las últimas décadas.

b) El segundo aspecto que es necesario considerar podríamos denominarlo de factor exógeno y se relaciona con el proceso de glo- 
balización que hoy ocurre a nivel planetario, pero que tuvo su origen en los países desarroIlados. De hecho, se trata de un factor exógeno en la medida que los estados I atinoamericanos no tuvieron influencia en su surgimiento, ni la tienen en los rumbos de la globalización, al menos hasta el momento. Lo que se puede observar al respecto es un acoplamiento reciente a las expectativas mundiales en torno de este proceso, sufriendo América Latina las consecuencias de adaptación y distante de recrear nuevas formas de comprensión y de actuación que partan de las realidades nacionales o continentales. A no ser la acomodación al proceso de globalización a través de la formación de bloques regionales que permitan la integración económica de modo a aprovechar las ventajas comparativas entre los países de la región, tales como Mercosul e Alca.

Tal vez un enfoque más específico o propio de los estados latinoamericanos ayudaría a rencauzamientos y/o readecuaciones del proceso de globalización y acrecentaría alguna originalidad saludable a las políticas gubernamentales y a los acuerdos entre los países latinoamericanos. Las preguntas que, a mí entender, deberían ser consideradas serían: ¿Es la misma cosa el proceso de globalización que la globalización de la economía? ¿Cómo pensar la cuestión nacional en el marco de la globalización? ¿Las políticas sociales, en cuanto derecho de todo ciudadano al bienestar y, consecuentemente, obligación del Estado de proveerlas, son incompatibles con la globalización? ¿O se trata meramente de una visión economicista de este proceso, pero no necesariamentela única?

Consideraciones sobre las reformas de la seguridad social y de los sistemas de salud

Las reformas de la seguridad social y de los sistemas de salud que en algunos países estan hoy siendo ensayadas, y que en otros se encuentran en su fase terminal, son guiadas por la restricción del gasto más que por la decisión de readecuar los sistemas a las necesidades en curso. No quiero decir que las necesidades no estén consideradas, lo están, aunque en un segundo plano, o sea, a posteriori de la definición del cuantum de recursos a ser alojados en el área social; en este sentido, Ias políticas son condicionadas a los recursos. Es importante señalar que ésta no es una cuestión secundaria, ya que tenerla en cuenta, al reflexionar sobre las reformas o al planificarlas, podría facilitar la incorporación de alternativas político-admi- nistrativo-financieras, en la lógica de operar los sistemas de salud de manera a al canzar una mayor eficiencia y efectividad de los mismos, en lugar de acrecentar inequidades en el usufructo de la salud.

Si pasamos ahora a considerar las reformas de los sistemas de salud en las décadas de los 80 y 90, podremos constatar que éstas fueron implementadas de acuerdo a parámetros de racionalización financiera y administrativa, salvo escasas excepciones en Europa y en América Latina, de las cuales apenas pueden mencionarse España y Brasil. Estas reformas ocurrieron en otro contexto político-social y económico; en Italia, la reforma se produjo a fines de la década de los 60, en el marco del Welfare State europeo. En el caso de Brasil, aunque fue posterior, a mediados de los 70 , la transformación del sistema de salud nace con la crisis del régimen militar y las reivindicaciones sociales por una liberalización política, junto a mejores condiciones de vida y especialmente de salud para la población.

En ambos casos se produjeron transformaciones de la base de sustentación de los sistemas de salud relativas a la propia concepción de la salud, a un cambio de perspectiva en la visión de la relación entre el Estado y la sociedad sobre la óptica de los derechos de ciudadanía y a transformaciones en las modalidades de gestión de los servicios, producción, atención y financiamiento de los mismos. La preponderancia del sector público de la salud, el acceso universal a los servicios, la descentralización, la participación social, el control social de los servicios por parte de la población y el pago indirecto de los mismos fueron los parámetros norteadores de estas reformas. Éstas tuvieron origen en la sociedad y no consiguieron avances significativos en su implementación debido, fundamentalmente, a las tendencias identificadas arriba en relación al carácter del Estado.

Contrariamente, las reformas racional izadoras que se originaron en Inglaterra y Estados Unidos fueron incentivadas por los gobiernos conservadores de Thatcher, en Inglaterra, y Reagan, en Estados Unidos, auspiciados por las crisis económicas de los 70 y de los 80 . En este contexto, las reformas de los sistemas de salud defendían la introducción de mecanismos concurrentes que incitasen al libre juego del mercado. Resumidamente, podriamos enumerar algunas de estas reformas que ocurrieron principalmente en Europa y en los Estados Unidos, sin pretender mencionarlas todas, mas, sobre todo, con el objetivo de ejemplificar los aspectos racionalizadores que las caracterizan. 
El "ticket moderador", que básicamente es un mecanismo de coparticipación del paciente en los costos de los servicios. La asistencia médica administrada, que busca controlar la utilización de los servicios a través de la oferta y de la demanda; en la cual los médicos son inducidos a prescribir los "servicios necesarios" y los pacientes ven restringidas las opciones de elegir el servicio y el profesional que los atenderá. La competición administrada que se sustenta en la creación de un sistema nacional de seguro de salud basado en la "libre" elección de consumidores concientes y en la competición de precios entre planes alternativos. El modelo de competición pública que no engendra un cambio radical en la estructura pública del sistema de sal ud, mas promueve la eficiencia interna a través de la introducción de incentivos de mercado en el sector público de la salud.

En los años 90, en los países de América Latina, propuestas de reformas de los sistemas de salud se apoyan en la constitución de sistemas mixtos público/privados, con aranceles diferenciados y libre elección de los servicios por parte de los usuarios. Para las poblaciones que están por debajo del nivel de subsistencia, caberian intervenciones focalizadas intermediadas por paquetes básicos de servicios de salud. En este sentido, la segmentación de las acciones de salud refuerzan la exclusión social, en la medida que la atención pasa a ser diferenciada por categorías sociales.

Al mismo tiempo, cualquier acción focalizada crea su propia demanda, como varios autores han comprobado al analizar la crisis de los Welfares States europeos, en los cuales acciones sociales para categorías sociales restrictivas acaban multiplicando recursos administrativos y financieros. Este tipo de acciones contribuye, también, a la corrosión de la solidaridad social, sustentadora de los principios del Welfare, ya que el conjunto de la sociedad no se vé beneficiado con intervenciones direccionadas a grupos poblacionales específicos y, en cambio, es afectado por impuestos crecientes sustentadores de estas acciones.

Mas, principalmente, reformas de este tipo, al quebrar con la universalidad en el usufructo del derecho a la salud, poseen un sentido contrario al carácter de inclusión de las políticas sociales, en las cuales el derecho inalienable al bienestar se constituye en un patrimonio de la sociedad como un todo.

En la medida que estas propuestas liberalizantes ganaron espacio en América Latina, apoyadas por las agencias internacionales, donde, específicamente, el Banco Mundial y el BID (Banco Interamericano de Desenvolvimiento) tuvieron un papel singular, las reformas de los sistemas de salud, que consideraron la salud en cuanto derecho universal de los ciudadanos y un deber del Estado el proveerla - y cuya expresión más desarrollada la encontramos en el caso de la llamada "reforma sanitaria" en Brasil - sufrieron serios reveses. La implementación de estas políticas exigía la valorización y la readecuación del sector público como prestador principal de servicios, asi como los recursos financieros necesarios para alcanzar beneficios de salud para toda la población, otorgando al Estado un papel principal y diferenciado en este proceso.

La implementación de reformas de esta naturaleza permite su efectivización en el campo de la acción política, en la medida que negociaciones y acuerdos entre diferentes actores e intereses afectados se tornan mecanismos insustituibles en la conducción de un proceso que, sin lugar a dudas, es eminentemente político. Nos referimos a la construcción de un sistema unificado de salud bajo la órbita del Estado que contemple la cobertura de salud universal de la población, operacionalizado a través de la descentralización de los servicios de atención a la salud en las provincias y municipios, con niveles jerarquizados en las funciones de sal ud - sean preventivas o curativas - , con un comando único localizado en el nivel central del sistema y recursos financieros definidos por ley.

Lo que ocurrió es que los avances en el terreno jurídico constitucional no tuvieron correspondencia con la ejecución de la política y esto fundamentalmente por la manera en que el gobierno, en el caso brasileño, actuó para obstaculizar el buen encaminamiento de las reformas. Lo que se puede observar en el nivel federal, instancia decisoria central de la implementación de la reforma dal sistema de salud (Gerschman, 1995), es el papel secundario adjudicado a la política de salud que se expresó en la ausencia del papel regulador del Ministerio de la Salud y del Consejo Nacional de Salud, órgano de representación de la sociedad en la implementación de la política de salud.

La ausencia de un Plano Nacional de Salud que contuviese las directrices básicas para guiar la efectivación del sistema único de salud, descentralizado y con un comando único federal , llevó a una descentralización administrativa del sistema de salud, en la cual no existían incentivos políticos ni financieros para una efectiva autonomía política y administrativa de los municipios. Por un lado, apesar del proceso de descentralización, la esfera federal continuaba adjudicando recursos para las es- 
feras provincial y municipal, como si estas esferas de gobierno fuesen simplemente prestadoras de servicios. Por otro lado, vez tras otra, los recursos dejaban de ser repasados por el gobierno federal o, si éste lo hacia, los atrasos en los repartos eran tan grandes que el sistema entraba en falencia.

De esta manera, la omisión del papel regulador central, por parte del Estado, que se fundaba en los presupuestos neoliberales, que regían la conducción de la política nacional, entraban en colisión con las reformas progresivas del sistema de salud, definidas constitucionalmente en 1988. De cualquier manera, cabe resal tar que, aunque los embates al Sistema Único de Salud fueron significativos y, en el período que va de comienzos de la década hasta la mitad de los 90, creció el sector privado, no se consiguió el desmantelamiento del mismo, debido al grado de articulación y resistencia propia del sector de salud.

Pensando alternativas políticas en el campo de la política social y de las políticas de salud

Tomando en consideración la discusión efectuada arriba, la última parte de este trabajo tendrá por objetivo enfrentar las dificultades que las políticas sociales presentan en el contexto actual, trayendo al centro del debate un mapeamiento de las alternativas posibles para el desarroll o de políticas sociales y, dentro de éstas, específicamente, de las políticas de salud en cuanto instrumentos generadores de equidad y de justicia social.

En los países del tercer mundo, se observa que los resultados de las políticas de ajuste económico incidieron negativamente en la pobreza estructural y en la marginalidad social, al generar desempleo creciente y un mercado informal de trabajo asociado a actividades ilícitas, tales como contrabando, robo, comercialización de drogas, prostitución, etc., en franca competición con el trabajo formal. Al mismo tiempo que el Estado se retrae de su función de regulación económica y de intervención en el campo social, estas funciones se tornan cada vez más necesarias e imprescindibles para la preservación del tejido social. Las cuestiones relativas a la pobreza estructural de estos países, en décadas pasadas, quedaban restrictas a las fronteras nacionales; en las últimas décadas, la pobreza y las actividades ilícitas se internacionalizaron, debido a los flujos migratorios de los países pobres para los países "ricos", creando también, en estos últimos, bolsones de pobreza, y debido también a que una parte significativa de las economías internacionales se sustentan sobre los ingresos de actividades ilícitas.

En los países europeos que desarrollaron poderosos Estados de Bienestar fundados en la ciudadanía plena y apoyados en políticas sociales concebidas como derechos adquiridos por ley, con la crisis de los años 80 y 90 , el Estado se retrae de sus funciones de proveedor, al tiempo que la pobreza exportada de los países subdesarrollados, sumada a la pobreza generada por el crecimento del desempleo y la deslegitimación del Welfare, genera profundas disrupciones en la base solidaria sobre la cual se fundamentan los Estados de Bienestar, afectando profundamente la propia idea de irrenunciabilidad de los derechos de ciudadanía. Por este camino, las políticas sociales sufrieron, en estas dos décadas, retrocesos considerables.

Sin embargo, a partir de la mitad de los 90 y como resultado de las políticas económicas de ajuste, los países industrializados de Europa, América y Asia entran en una crisis social y política tan aguda que la misma incita a una retomada filosófico/ética sobre los valores que permean la existencia y la sobrevivencia humana en el fin de este siglo, que no pueden ser reducidos a un "enfoque unidimensional de la economía". En este contexto, resurgen valores de solidaridad social y la necesidad de volver a pensar la ciudadanía plena como conquista irrenunciable de las sociedades y Estados modernos.

En este sentido, vale la pena aproximar al debate de las políticas sociales alternativas conceptuales, para pensar la sociedad y la política en el fin del milenio, junto a al ternativas concretas para el retorno de la ciudanía social. Algunas consideraciones al respecto de la vida en sociedad y del ejercicio de la política en las democracias de fin del milenio podrían aproximarnos a recrear instrumentos conceptuales más apropiados para la comprensión de los procesos de desapropiación de las identidades individuales, sociales e históricas.

Las últimas décadas se caracterizaron por una profunda desintegración del tejido social. Esta desintegración recuerda la idea durkheimiana de la anomía social como una característica constitutiva de aquell as sociedades que manifiestan una pérdida en la normatividad y en la regulación de la vida social. El ejercício de la política se agota en democracias "procedurales", en las cuales no existen formas de participación y de representación política de la sociedad fuera de la elección de autoridades gu- 
bernamentales. Los partidos se transformaron en máquinas electorales bien aceitadas por marketing, dinero y medios de comunicación; órganos extraños a las necesidades sociales, en los cuales las personas no creen, excepto por los beneficios personales - dinero y poder que los políticos obtienen a través de los cargos partidarios.

La privación social, ocasionada por el desempleo creciente y por omisión del Estado, profundiza todavía más las brechas existentes en la sociedad. La pérdida del sentimiento de pertenencia y de identidad social refuerza el individualismo, que impide la formación de identidades colectivas más amplias. Sin embargo, otras tendencias comienzan a proliferar, ocupando espacios sociales y políticos subjetivos, que Giddens (1991) caracteriza como "política de vida", que trae al escenario político problemas ligados con cuestiones fil osóficas, de derechos humanos y de moralidad.

La política de vida presume un cierto nivel de emancipación, tanto de la tradición cuanto de la dominación jerárquica. Guiddens define la política emancipatoria como una perspectiva general que se ocupa fundamental mente de la liberación de los individuos y grupos de aquell os impedimentos que afectan negativamente las oportunidades de vida de las personas. En este sentido, la política de vida envuelve derechos y obligaciones, y el Estado continúa siendo la principal instancia administrativa dentro de la cual estas cuestiones pueden ser solucionadas dentro de la ley.

Consecuentemente, en el ámbito de las políticas sociales, interesa recuperar la relación entre la expansión y la difusión de las políticas sociales con la existencia de los derechos a una ciudadanía social plena. La efectivación de políticas sociales de carácter universal profundizó y afianzó el papel central del Estado en la atenuación de las diferencias sociales, al instaurar un proceso de redistribuición de rentas que tuvo como destinatario al conjunto de la población. Esta cuestión, prácticamente abandonada durante las dos últimas décadas, fue transformada en asunto secundario en la medida que, para el neoliberalismo, la pobreza es un estímulo para el trabajo y los individuos deben responsabilizarse por su propio bienestar, retirando al Estado de estas funciones.

Cabe así, en esta última parte del trabajo, traer para el debate de las políticas sociales alternativas que retomen valores morales que apunten para la recuperación de condiciones dignas de existencia humana sustentadas sobre los principios de una ciudadanía plena universal. La propuesta en cuestión es denomina- da de renta de ciudadanía o ingreso ciudadano, y comienza a ser discutida en varios países; aunque todavía se mantiene al interior del debate académico, parece una alternativa de política social extremadamente interesante, que valdría la pena considerar, en la medida que preserva y recrea valores de solidaridad social. Paralelamente, restituye la esperanza de aproximarnos, vía políticas sociales, a una reforma del Estado, de manera a tornarlo eficiente y transparente a la sociedad, como veremos a continuación.

La alternativa del ingreso de ciudadanía tuvo su origen en Europa, en la década de los 90 , siendo sus mentores pensadores del campo de la política, de la economía y de la filosofía, como Van Parjis (1994), Gorz (1994), Bresson (1994), Euzeby (1993), Offe (1992), entre otros. En las palabras de Gorz, que expresan el sentido de esta discusión en los autores mencionados:

"La garantía deun ingreso deexistencia puede cumplir una función adaptada a la evolución de soci edades avanzadas que poseen un volúmen creciente de riquezas con un volúmen decreciente de trabajo...La renda de existencia tendría una doblefunción: compensaría el de sempleo total o parcial y sería una iniciación al trabajo intermitente, de horario variable,atendiendo a la demanda de las empresas... En fin y sobre todo, el derecho al ingreso sería incondi cionalmente y evi dentemente desconectado de la obligación detrabajar dentro dela esfera económica o de asumir una función considerada util económi camente... se pasaría de una socie dad de pleno empleo a una sociedad de plena actividad, a tra vés de actividades microsociales que articulen eintegren la comunidad, desempeñando éstas un papel de convivencia social quecontribuiría para el enriquecimi ento dela vida; reconoci éndose en estas actividades una importancia y dignidad social igual a aquélas del mundo del trabajo" (Gorz, 1994:51).

De una parte, el “ingreso ciudadano" busca establecer un sistema integrado de transferencias fiscales e impuestos, pretendiéndose obtener la mayor redistribución posible de ingresos en una dirección que va de los sectores de altos ingresos para los más pobres. Por otra parte, se trata de una propuesta compatible con la preservación de otras instituciones de política social, particularmente si no se está en condiciones de garantizar a través del ingreso ciudadano un nivel pleno de satisfacción de todas las necesidades esenciales.

Con la intención de distinguir las diferencias existentes entre renta mínima y renta ciudadana, es necesario considerar que la primera se originó dentro de la concepción neoliberal, 
en cuanto la propuesta de renta ciudadana surge como una alternativa de profundizar y legitimar las políticas sociales. La idea de renta mínima fue levantada por Milton Friedman (Friedman \& Friedman, 1987) y propone la adjudicación para aquellas personas que están por debajo de la línea de subsistencia de un ingreso muy reducido, de forma tal de que éste sea un estímulo para buscar cualquier ingreso adicional, sin transformaciones de peso en la estructura tributaria. La utilización de la idea de renta mínima que ha sido hecha en América Latina, excepto algunas excepciones local es en las cuales se la aplicó de manera complementaria al sistema de seguridad social, llevó a un desmantelamiento de los componentes universales del sistema de políticas sociales, ya que fue encarada como un programa encaminado a la población indigente, resultando en un achicamiento de la cobertura de los sistemas de seguro social. Genera mayor regresividad tributaria, si tenemos en cuenta que deduce impuestos quién declara y quién declara es quien gana más.

Contrariamente a lo expuesto arriba, el ingreso ciudadano se constituiría como un derecho de carácter universal. Todos los ciudadanos podrían usufructuar de este ingreso de ciudadanía, sin la exigencia de responder por cualquier contraprestación a cambio del mismo. En este sentido, el ingreso ciudadano estimularía actividades que son socialmente útiles (servicios sociales) y que merecen ser remuneradas a través de las riquezas generadas socialmente. En relación a los mecanismos para la operacionalización del ingreso ciudadano: 1) no requiere test de medios, porque se paga a todos; 2) es garantizado previamente para toda la población; en este sentido, cumple una función preventiva y no curativa; 3) define un piso sobre el cual las personas pueden acumular cualquier otro ingreso.

Las unidades de tributo y de beneficio deben ser las mismas, y la implementación de un ingreso ciudadano no podría estimular a que las personas adopten un determinado arreglo familiar para recibirlo ya que el mismo es dado al individuo y no a la familia. Respecto al monto del ingreso ciudadano, se buscaría que éste tuviese un nivel tan elevado como lo permita la riqueza de cada sociedad.

En lo que se refiere a la relación entre el ingreso ciudadano yel pago de impuestos, se prevee que el monto de dinero recibido en calidad de ingreso ciudadano no pagaría impuesto. Habría un punto de inflexión entre ingreso y tributación en el cual no habria tampoco tasación del ingreso total del ciudadano, en el caso que éste fuese apenas una cifra ligeramente mayor que el nivel del ingreso ciudadano. Por encima de este punto, la persona pagaría impuestos proporcionales al ingreso obtenido. Cabe señalar que diferentes autores proponen alternativas variadas en relación a las posibilidades de instituir reformas tributarias que sustenten la política social de ingreso ciudadano, mas como ésta es una discusión más técnica que conceptual no será tratada aquí.

Considerando ahora la política de salud en cuanto política social, los principios a ser instituidos, o mantenidos, en aquellos países que desarrollaron procesos transformadores en el sector de la salud, deberían contemplar la salud de la población como resultado de las condiciones de vida de la sociedad. Así, la salud, más que la atención a la enfermedad, es la capacidad que una sociedad tiene de evitar la enfermedad. Una sociedad saludable es aquélla que ofrece vivienda, infraestructura cloacal y eléctrica, gas, alimentación, trabajo, educación y lazer para todos sus miembros. Una sociedad en que la salud, en un sentido amplio, es definida como un derecho de ciudadanía y un deber del Estado de proveerla para todos.

Pero, aún defendiendo la salud como un derecho universal, no se puede olvidar que los procesos de transformación social y los adelantos tecnológicos que se operaron en este siglo, en el campo de la medicina, como en tantos otros, llevaron, de una parte, a una mayor longevidad de las personas y, de otra, a la presencia de enfermedades antes desconocidas y de tratamientos extremadamente costosos, que exigen una readecuación del sector de la salud, de manera a mantener el derecho universal a la salud, interfiriendo sobre el crecimiento de los costos crecientes del sector. Dos cuestiones aquí me parecen de fundamental importancia. La primera se refiere a una reflexión ética respecto de los avances de la medicina, en los cuales inversiones enormes son realizadas, no con la finalidad de una vida sal udable y una existencia digna para la raza humana, mas colocando la vida como una mercancía a disposición de quién pueda comprarla. Esto significa que campos de conocimiento como la biotecnología, que puede producir desde inmunobiológi cos hasta armas biológicas, o crear vida artificialmente, o replicar ovejas y - ¿por qué no? - seres humanos, algún dia dependen dela existencia de un mercado en el cual los procedimientos tecnológicos tienen un valor monetario, independientemente de su valor moral mejorar la vida humana en el planeta. Consecuentemente, los costos crecientes hacen con que las intervenciones de alta complejidad 
sean utilizadas solamente por quién tiene medios económicos para pagarlas, en cuanto, cada vez más, sectores importantes de la sociedad mueren de enfermedades que pueden ser prevenidas y que requieren intervenciones relativamente simples.

Llevar en consideración estas cuestiones implicaría reconsiderar la idea del derecho universal a la salud, ya no en términos formales de la definición del derecho universal a la salud, mas desde la perspectiva del efectivo usufructo del derecho a la salud. Así, la manutención del derecho universal a la salud no significaría usufructuar gratuitamente de los beneficios de manera homogénea para toda la población.

De la misma manera que la renta ciudadana define un límite de ingresos a partir del cual las personas deben pagar impuestos dependiendo del nivel de ingresos que posean, las políticas de salud deberían incentivar un comportamiento progresivo en la relación entre aportes y beneficios. O sea, en relación a la salud, el derecho a la salud debería estar relacionado al "ingreso ciudadano", definiéndose una cuota igual de beneficios de salud para todos los ciudadanos y para aquellos que precisacen usufructuar de ben eficios que requieren intervenciones costosas; funcionaría el principio de solidaridad. No paga quién tiene por único ingreso la "renda de ciudadanía", y quién tiene un ingreso mayor pagará honorarios al sector pú-

\section{Referencias}

BRESSON, Y., 1994. Le Partage du Temps et des Revenues. Paris: Economica.

CHESNAIS, F., 1997. Mundialização do Capital, regime de acumulação predominantemente financeira e programa de ruptura com o Neoliberalismo. Revista da SociedadeBrasileira de Economia Política, 1:7-34.

DELCOURT, J., 1992. Globalisation de l'économie et progrès social. L'Etat social à I'heure de la mondialisation. Futuribles, 156:3-34.

DINIZ, E., 1997. Crise, governabilidade e reforma do Estado: em busca de um novo paradigma. In: A Miragem da Pós-Modernidade. Democracia e Políticas Sociais no Contexto da Globalização (S. Gerschman \& M. L. V. Werneck, org.), pp. 115-124, Rio de Janeiro: Editora Fiocruz.

EUZEBY, C., 1993. Du Revenue Minimum d'Insertion au Revenue d'Existence. Futuribles, 177:15-27.

GIDDENS, A., 1991. Modernity and Self Identity Self and Society in the Latin Modern Age. Stanford: Stanford University Press. blico de la salud, cuyo cálculo dependerá de cuánto su ingreso está por encima de la "renda de ciudadanía". Al mismo tiempo, se mantendría la libertad de elección por parte del usuario para contratar un seguro privado de salud, pero se exigiría de los seguros privados de salud el pago de un impuesto relacionado a la utilización de producción de tecnología e investigación originadas en las instituciones públicas de ciencia y tecnología y de un impuesto a ser pago por los usuarios de seguros privados al sector público de la salud, independientemente de la utilización de los servicios públicos.

Las alternativas aquí colocadas no tuvieron la intención de presentar una fórmula a través de la cual los problemas analizados se resolverían, pero, sobretodo, la intención de reflexionar sobre las condiciones que podrían contribuir al renacimiento de la ciudadanía plena. Seguramente, implementar una propuesta tan compleja como ésta generaría muchos problemas que ninguna fórmula podría anticipar. Mi intención fue, exclusivamente, la de cuestionar visiones unilaterales y comprovadamente falladas para enfrentar los problemas comunes que hoy asolan las sociedades y estados latinoamericanos; presentando perspectivas que, aunque todavía no fueron probadas, expresan un pensamiento direccionado a crear condiciones de existencia más justas, solidarias y equitativas para la sociedad en su conjunto.
GORZ, A., 1994. Revenue Minimum et Citoyenneté, Droit au Travail vs. Droit au Revenue. Futuribles, 184:49-60.

GRINDLE, M. S. \& THOMAS, J. W., 1993. Policy makers, policy choices and policy outcomes: political economy of reform in developing countries, In: Reforming Economic Si stemsin Devel oping Countries (D. Perkins \& M. Roemer, eds.), pp. 74-91, Boston: Harvard University Press.

OFFE, C., 1992. A non productivist design for social policies. In: Arguing for Basic Income, Ethical Fundations for a Radical Reform (P. Van Parjis, ed.), pp. 32-58, London: Verso.

SILVA, E., 1993. Capitalist coalitions, the State and neoliberal economic restructuring, Chile 1973-88. World Politics, 45:45.

VAN PARJIS, P., 1994. Au delà de la solidarité. Les fondements éthiques de L'Etat providence et son dépassement. Futuribles, 184:5-29. 